\title{
Cosmic X-ray bursts
}

\author{
from Kenneth Brecher
}

Cosmic X-ray bursts, first observed by the Russian Cosmos 428 satellite in 1971 and reported in a widely overlooked paper by O. P. Babushkina et al. (Sov. Astron. Lett., 1, 32; 1975) have now been detected by many other X-ray satellites. The ANS, SAS 3, Vela 5, and Ariel V groups have reported in rapid fire succession a range of exciting and perplexing observations of these fast transient cosmic events. In this issue of Nature, (page 562) J. Heise et al. report on their observations of "rapidly repetitive Xray burster" MXB 1730-335 made with the Astronomical Netherlands Satellite. This object, discovered by the Massachusetts Institute of Technology SAS 3 group and reported by Professor Walter Lewin (IAU Circ., 2922, March 5, 1976) is perhaps the most enigmatic "X-ray burster" found to date. Sometimes bursting at severalsecond intervals, at other times waiting minutes between events, its X-ray burst pattern reminds one very much of a relaxation oscillator. Indeed, as pointed out by Professor George Clark (MIT) the tight correlation found in the SAS 3 data between the X-ray burst size and the time to the following burst is reminiscent of the behaviour of a neon light bulb (rather than, for example, a flush toilet). It is as if a reservoir of energy (or material to provide it) must build up to a fixed level and discharge; the larger the discharge, the longer it takes to build up for the next firing. (In the flush toilet analogue, with filling occurring at a constant rate, the size of a burst would be proportional to the time preceding it.)

What astrophysical object could produce such behaviour? The ANS group report a position for the $\mathrm{X}$-ray burster which they intersect with the SAS 3 error box: the overlap region contains what according to W. Liller (Harvard College Observatory) may be a globular cluster (a stellar system several light years across consisting of $10^{4}-10^{6}$ stars). The importance of the possible association of the rapid burster with a globular cluster cannot be overemphasised: it may be something specific to these stellar systems as a whole, rather than the individual stars within them, which gives rise to the observed X-ray bursts. Heise et al. point out that at least three bursters appear to be associated with globular clusters. Nonetheless, it should be noted that at least four burst sources reported by the MIT SAS 3 group-MXB 1728-34, a source in the Galactic anti-centre region, one in Puppis, and another in Aquilla ( $I A U$ Circ., 2922, March 5, 1976, and private communications from $W$. Lewin)-are not obviously associated with globular clusters, or, if they are, there is no clear reason why the optical counterparts are so obscured as to be utterly unobservable. It is truly remarkable that of the 100 or so Galactic globular clusters known in the halo population the unobscured ones are not X-ray bursters, but that it is claimed that several of the obscured ones are. Perhaps a second hitherto unsuspected disk population of obscured globular clusters, or ones containing invisible stars, houses these bursters.

Making the bold assumption that it is in the nature of globular clusters to burst out occasionally, what is it specifically that gives rise to the $\mathrm{X}$-ray bursts? It has been suggested for some time that a globular cluster could become unstable to the formation of a very dense or tightly bound stellar configuration at its centre. Taking this view one step further, J. Bahcall and J. Ostriker (Princeton University) and, independently, J. Arons and J. Silk (University of California, Berkeley) proposed that the steady X-ray emission observed from five globular clusters arises from accretion of intracluster gas on to a central, massive $\left(10^{2}-10^{3} M \odot\right)$ black hole. Adapting rapidly to the new observations, Bahcall and Ostriker have now suggested that these same black holes can also emit X-ray bursts, provided that a neutron star were orbiting the hole. Postulating an accretion disk of gas around the black hole, the neutron star would then periodically crash through the disk, slurp up some gas, and belch out the observed X-rays. This model, offered as an explanation for the recurrent X-ray burst source apparently associated with the steady X-ray source 3 U $1820-30$ and possibly located at the centre of the globular cluster NGC6624, is difficult to reconcile with the very brief time interval between bursts from the rapid burster. Indeed, the change in mean burst period from $0.18 \mathrm{~d}$ (Clark, IAU Circ., 2907) to $0.12 \mathrm{~d}$ (Clark, IAU Circ., 2932) makes this model suspect even for NGC6624. The suggestion by J. Grindlay and $H$. Gursky (Center for Astrophysics) that the pulse shape of the X-ray bursts could arise from the scattering of a sharp X-ray pulse on a spherical cloud of very hot gas (rather than a disk) which is held down by a massive black hole is at variance with the above suggestion. As shown in detailed Monte Carlo calculations by C. Canizares (MIT), however, the shape of the decay phase of the X-ray bursts can be well described by Compton scattering of the photons on cooler gas surrounding an object of a few solar masses rather than a massive black hole. Unless one devises one model for each source, a procedure which seems premature in view of the remarkable similarity in pulse structure from one burst source to another, one must turn from black holes to some other, more physical and realistic, model.

Most optically identified discrete Galactic X-ray sources found to date have been associated with mass exchange binary star systems, probably containing as one member a collapsed object (neutron star, white dwarf, or black hole). Various authors, such as J. Arons and S. Lea (Berkeley), have suggested that instabilities in accretion flow on to the collapsed object could give rise to rapidly variable $X$-ray fluxes from these objects. W. Baan (MIT) has suggested that accretion disk instabilities occurring around single neutron stars accumulating gas while moving through globular clusters could account for many of the particular properties observed in MXB $1730-335$. Flash nuclear burning on the surface of a neutron star has been proposed as an X-ray burst model by $\mathrm{L}$. Maraschi (Milan) and A. Cavaliere (Frascati). Other models, such as the stellar lightning model offered by Rrose Selavy (Zurich) have not been worked out in sufficient detail to make a direct comparison with observations possible at present.

Despite the fact that in the past few years black holes have been offered up as a panacea for explaining everything from the Tunguska event, to the absence of solar neutrinos, to the energy emitted by quasars, and even as a solution to the terrestrial energy crisis, there seems to be little direct observational support for the assertion that X-ray bursters provide evidence for the existence of massive black holes. If it turns out that the X-ray bursts arise as the result of one more complication in mass exchanging binary star systems, they will almost certainly lose some of their current fascination and mystery. Still, it must be said that it is really too early to tell whether the X-ray bursts are the astronomical discovery of the decade, or ffiust a flash in the pan. 